\section{Ätherische Öle und Riechstoffe}

In diesem sehr schön aufgemachten Buch erfährt der Leser einiges über die Geschichte der Duftstoffe, über die Natur von Riech- und Geschmacksstoffen sowie von Duftpflanzen und Duftnoten.

Das Werk ist übersichtlich gegliedert. Über 250 Duftpflanzen werden einzeln vorgestellt: Man erhält Informationen zur taxonomischen Klassifikation, zum Vorkommen und zur Verbreitung, zum Anbau sowie zu den duftstoffhaltigen Pflanzenteilen. Jede Pflanzenbeschreibung ist mit einem farbigen Bild illustriert. Einem kurzen Kapitel über Balsame, Gummiharze und Harze folgen detaillierte Erläuterungen $\mathrm{zu}$ ätherischen Ölen sowie schließlich in einem ausführlichen $\mathrm{Ka}$ pitel die chemischen Charakteristika von bekannten Riechstoffen.

Das Werk ist mit einem ausführlichen Literaturverzeichnis und einem hervorragenden Register versehen. Es ist ein Schatz nicht nur für Apotheker, Kosmetik- und Duftstoffhersteller, Naturstoffchemiker und Ärzte, sondern für alle, die sich für Pflanzen und Naturstoffe interessieren.

\section{J. Ring} Rotz, Kormann: Duftpflanzen - Pflanzendüfte. Ätherische DM 128,-. ISBN: 3-609-65140-7.

\section{Pollenkalender}

Der „Pollenkalender“ von Erika Stix liegt nunmehr in der 2. Auflage vor. Wiedergegeben werden die Ergebnisse von zum Teil mehrjährigen Pollenanalysen in verschiedenen $\mathrm{Re}$ gionen Deutschlands. Mit BurkhardPollenfallen wurden in der Regel von Februar bis Oktober insgesamt 75 Pollentypen erfaßt. Die Fallen standen in neun verschiedenen Biotopen, wie z.B. in einem Bergwald, einem Stadtzentrum, einer Wiese oder in einer Küstendüne.

Für jeden der neun Standorte erfolgt in dem Buch eine kalendarische Übersicht des Fluges der häufigsten
Pollenarten. In der jeweiligen Graphik werden die Mittelwerte der in den Jahren ermittelten Pollenzahlen übersichtlich wiedergegeben. Klimatisch bedingte Abweichungen im Pollenflug der einzelnen Jahre sind nicht berücksichtigt. Ein Vergleich der einzelnen Biotope zeigt dem Leser, daß der regionale Pollenflug deutliche qualitative und quantitative Unterschiede aufweist.

In einem abschließenden Kapitel „Zur Repräsentanz der Pollenflugkalender" vermittelt Frau Stix interessante Informationen zu den Flugeigenschaften verschiedener Pollen sowie auch zur Abhängigkeit des Pollenfluges von klimatischen und geographischen Faktoren.

Insgesamt stellt das Buch für den pollenallergischen Patienten eine anschauliche und interessante Informationsmöglichkeit über die Auslöser seiner Atemwegserkrankung dar.

\section{H. Müsken}

Stix: Pollenkalender. Wissenschaftliche Verlagsgesellschaft, 1997. 56 Seiten. DM 58,-. ISBN: 3-8047-1500-1.

\section{Chemikalien-}

\section{unverträglichkeit}

Überempfindlichkeitsreaktionen gegen Chemikalien stellen ein zunehmendes Problem in den allergologischen Praxen, aber auch im öffentlichen Bewußtsein dar. Es handelt sich dabei um Zustandsbilder mit polysomatischen Beschwerden meist subjektiver Natur, die in klassische Krankheitsbilder oft schwer einzuordnen sind, häufig eine starke psychosomatische Überlagerung aufweisen und unter verschiedenen Namen diskutiert werden, wie z.B. „Öko-Syndrom“, „Multiorgan-Dysästhesie“, „,idiopathische Umwelt-Intoleranz", Multiple Chemical Sensitivity“ (MCS) u.a.m. Ein Hauptproblem in der wissenschaftlichen Diskussion ist die Tatsache, daß die Existenz einer tatsächlichen neuen Krankheit immer noch kontrovers diskutiert wird.

Die Autoren gehen davon aus, daß es sich hier wirklich um eine neue

\section{Neurodermitis}

In diesem handlichen Buch gelingt es dem Autor, auf 150 Seiten das Wesentliche über die Neurodermitis allgemeinverständlich darzustellen - unter kritischer Würdigung von alternativen Methoden.

Das Buch ist logisch aufgebaut und sehr übersichtlich gestaltet. Kurze Zwischenüberschriften am breiten Rand erleichtern das schnelle Lesen. Wichtige Merksätze sind graphisch hervorgehoben. Viele Kapitelüberschriften sind als Fragen formuliert, die dann auch beantwortet werden.

Das Buch steht ganz auf dem Boden der wissenschaftlichen Medizin und setzt sich vorurteilsfrei mit den alternativen Behandlungsmöglichkeiten einschließlich Candida, Bioresonanz etc. auseinander. Es ist lesenswert für Patienten und Ärzte.

J. Ring

Tüttenberg: Neurodermitis. Ullstein-Verlag 1997. 158 Seiten. DM 29,90. ISBN: 3-333-01005-4.

Krankheit handelt, und beschreiben den aktuellen Wissensstand aus ihrer Sicht, die vom Gedankengut der ,Klinischen Ökologie" geprägt ist.

Das Buch ist folgendermaßen gegliedert: Begriffsbestimmung, Symptome und klinische Befunde, empirische Untersuchungen, Erklärungen, Diagnose, Behandlung, Auslöser, Arbeitsschutz und Behandlungsmöglichkeiten sowie politische Perspektiven.

Leider ist zwischen wissenschaftlich gesicherten und nur vermuteten Aussagen kein für den Leser erkennbarer Trennstrich gezogen. Der wirkliche Kenntnisstand auf diesem hochproblematischen Gebiet wird aus dieser nicht nur formal zu unrecht als Handbuch bezeichneten Broschüre nicht ersichtlich.

\section{J. Ring}

Maschewsky: Handbuch Chemikalienunverträglichkeit (MCS). Medi-Verlag, Hamburg, 1996. DM 34,-- ISBN. 3-9803957-4-X 